\title{
Dynamical and gravitational instability of an oscillating-field dark energy and dark matter
}

\author{
Matthew C. Johnson* and Marc Kamionkowski ${ }^{+}$ \\ California Institute of Technology, Mail Code 130-33, Pasadena, California 91125, USA
}

(Received 22 May 2008; published 18 September 2008)

\begin{abstract}
Coherent oscillations of a scalar field can mimic the behavior of a perfect fluid with an equation-of-state parameter determined by the properties of the potential, possibly driving accelerated expansion in the early Universe (inflation) and/or in the Universe today (dark energy) or behaving as dark matter. We consider the growth of inhomogeneities in such a field, mapping the problem to that of two coupled anharmonic oscillators. We provide a simple physical argument that oscillating fields with a negative equation-of-state parameter possess a large-scale dynamical instability to growth of inhomogeneities. This instability renders these models unsuitable for explaining cosmic acceleration. We then consider the gravitational instability of oscillating fields in potentials that are close to, but not precisely, harmonic. We use these results to show that if axions make up the dark matter, then the small-scale cutoff in the matter power spectrum is around $10^{-15} M_{\oplus}$.
\end{abstract}

DOI: 10.1103/PhysRevD.78.063010

PACS numbers: 95.36.+x, 95.35.+d

\section{INTRODUCTION}

Observational evidence for inflation, for accelerated expansion in the current Universe [1], and for nonbaryonic dark matter has motivated the search for exotic forms of energy. Scalar fields have been thoroughly investigated for both pressureless and negative-pressure matter. If the kinetic and potential energies of the scalar field are nearly equal, then the field behaves as pressureless matter. The most important example is axion dark matter, which can be described as coherent oscillations of the axion field in a nearly-harmonic potential. Alternatively, if the ratio of potential energy to kinetic energy is sufficiently large, cosmic acceleration can be induced. Quintessence achieves this with a rolling scalar field, the rolling slowed by Hubble friction [2]. Spintessence $[3,4]$ achieves this with a complex scalar field rotating in an internal $U(1)$ symmetric potential; here, the centripetal acceleration, rather than Hubble friction, prevents the scalar field from falling directly to its minimum. A coherently oscillating scalar field can also drive cosmic acceleration.

Coherent oscillations in a harmonic potential behave as nonrelativistic matter, but oscillations in a more general potential can mimic a perfect fluid with an arbitrary equation-of-state parameter. For example, in the presence of a power-law potential $V(\phi) \propto|\phi|^{n}$, one finds that $w=$ $(n-2) /(n+2)$ [5]. The power-law index $n$ determines, through the virial theorem, how the kinetic- and potentialenergy densities are apportioned over one oscillation cycle. When $n<1$, we have $w<-1 / 3$, illustrating that coherent oscillations of a scalar field might drive a period of inflation in the early Universe [6-13] or provide a candidate for dark energy [14-20].

The purpose of this paper is to investigate the dynamical (i.e., those arising from the scalar-field dynamics) and

\footnotetext{
*mjohnson@theory.caltech.edu

${ }^{+}$kamion@tapir.caltech.edu
}

gravitational instabilities to the growth of inhomogeneities that may arise in oscillating-field matter. The presence of such inhomogeneities are important for determining the viability of these models for describing inflation, dark energy, and dark matter.

The question of dynamical stability of oscillating-field dark energy has been analyzed, in the context of inflation, in Refs. [21,22], concluding that oscillating fields that give rise to accelerated expansion are indeed unstable to the growth of inhomogeneities. This prior work analyzed the equations of motion, and it focused on the resonant growth of perturbations on small scales. Reference [23] considered further the possible nonlinear evolution of the field. References $[6,15,16]$ speculated that accelerating oscillating potentials may have a large-scale instability, but they did not carry out a full instability analysis. In spintessence, an analysis of the linearized equations of motion for scalarfield perturbations about the homogeneous solution shows that many models driving cosmic acceleration (for a power-law potential, large-scale instabilities set in for $n<$ 2) are dynamically unstable [3,24].

In addition, prior work $[25,26]$ has shown that gravitational instabilities of oscillations in a harmonic potential, suitable for oscillating-field dark matter, are suppressed on sufficiently small scales. This scale determines the smallscale cutoff in the matter power spectrum, and has potentially interesting implications for cosmology.

Our principle new contribution to the dynamical stability of oscillating-field matter is a simple physical picture of the criterion for stability at large scales. We show that the perturbed scalar-field equation of motion is identical to that of two coupled anharmonic oscillators. This picture then allows us to provide a simple understanding of why oscillating potentials with negative pressure should be unstable at large scales, while those with positive pressure should have large-scale stability. We then verify these conclusions analytically for potentials that are nearly harmonic (i.e., nearly pressureless) and numerically for a broader range of 
potentials. Our gravitational analysis generalizes prior work $[25,26]$ by considering potentials that are nearly, but not precisely, harmonic.

Below, we first review (in Sec. II) the homogeneous evolution of an oscillating scalar field. We then provide in Sec. III a heuristic discussion of the dynamical instability, beginning first with an explanation for the origin of the dynamical instability in spintessence models. Section IV shows analytically that for nearly-harmonic potentials, instability occurs for negative-pressure potentials and stability occurs for positive-pressure potentials. Section $\mathrm{V}$ verifies this conclusion numerically for more general potentials. Section VI includes gravity in the analysis and works out the gravitational-instability scale for nearly-harmonic potentials. We discuss here the application to axion dark-matter models, working out the smallscale cutoff in the matter power spectrum. Section VII presents some concluding remarks.

\section{HOMOGENEOUS EVOLUTION}

Consider a scalar field with potential $V(\phi)=V(-\phi)$ minimized at $V(\phi=0)=0$. The equation of motion for the scalar field is

$$
\ddot{\phi}+V^{\prime}(\phi)=0,
$$

where the dot denotes a derivative with respect to time $t$ and the prime denotes a derivative with respect to the scalar field $\phi$. The scalar field will undergo periodic motion in this potential, with a period

$$
T\left(\phi_{0}\right) \equiv \frac{2 \pi}{\omega\left(\phi_{0}\right)}=4 \int_{0}^{\phi_{0}} d \phi\left[V\left(\phi_{0}\right)-V(\phi)\right]^{-1 / 2},
$$

that depends, most generally, on the scalar-field amplitude $\phi_{0}$. The period can also be written in terms of the action [16]

$$
J=4 \int_{0}^{\phi_{0}} d \phi \sqrt{2\left[V\left(\phi_{0}\right)-V(\phi)\right]}
$$

as $T=d J / d V_{0}$, where $V_{0}=V\left(\phi_{0}\right)$.

For $V(\phi) \propto|\phi|^{n}$, the angular frequency $\omega\left(\phi_{0}\right) \propto$ $\left|\phi_{0}\right|^{(1 / 2)-(1 / n)}$. Thus, for $n=2$, the frequency is amplitude independent. For $n>2$, the frequency increases with amplitude, and for $n<2$, the frequency decreases with amplitude.

On time scales long compared with the oscillation period, the scalar-field oscillations behave like a perfect fluid with energy density $\rho$ and pressure $p$,

$$
\begin{gathered}
\rho \equiv\left\langle\dot{\phi}^{2} / 2+V\right\rangle, \\
p \equiv\left\langle\dot{\phi}^{2} / 2-V\right\rangle,
\end{gathered}
$$

where the angle brackets denote a time average. For this to hold, and to neglect the Hubble friction term in Eq. (1), we assume that the period of oscillations is much smaller than a Hubble time. The virial theorem tells us that $\left\langle\dot{\phi}^{2} / 2\right\rangle=$ $(1 / 2)(1+w) V_{0}$ and $\langle V(\phi)\rangle=(1 / 2)(1-w) V_{0}$, where $V_{0} \equiv V\left(\phi_{0}\right)$ and $w \equiv p / \rho=(n-2) /(n+2)$ is the equation-of-state parameter. These results can be summarized in terms of the action by [16]

$$
w=\frac{J}{V_{0}} \frac{1}{\left(\frac{d J}{d V_{0}}\right)}-1 .
$$

Differentiating the relation $T=d J / d V_{0}$ and rearranging algebraically, we obtain an expression for the variation of the frequency with field amplitude,

$$
\frac{d \omega}{d \phi_{0}}=\frac{\omega^{2}}{2 \pi(1+w)^{2}} \frac{d V\left(\phi_{0}\right)}{d \phi} \frac{J}{V_{0}}\left[\frac{d w}{d V_{0}}+\frac{w}{V_{0}}\right] .
$$

Thus, the frequency increases with amplitude unless $V_{0} d w / d V_{0}+w<0$.

If $w$ is independent of $\phi_{0}$, as for power-law potentials, then the frequency will decrease with amplitude when $w<$ 0 and increase with amplitude when $w>0$. If $w$ is allowed to change with $\phi_{0}$, then we can have $w<0$ and $d \omega / d \phi_{0} \leq 0$, but only temporarily.

There is also a nice geometric interpretation of the value of the equation-of-state parameter [6]. Using energy conservation and the field equation, we can write

$$
\left\langle V-\phi \frac{d V}{d \phi}\right\rangle=-(1+3 w) \frac{V_{0}}{2} \text {. }
$$

The left-hand side is the average of the intercept of the tangent to the potential, and is in general positive for convex (about $\phi=0$ ) potentials and negative for nonconvex potentials. A few simple example potentials are shown in Fig. 1 to illustrate this, although it is easy to imagine potentials with more complicated features. In general, to produce accelerated expansion, there must be a relatively flat region of the potential with positive energy somewhere along the oscillation cycle, according with the intuition

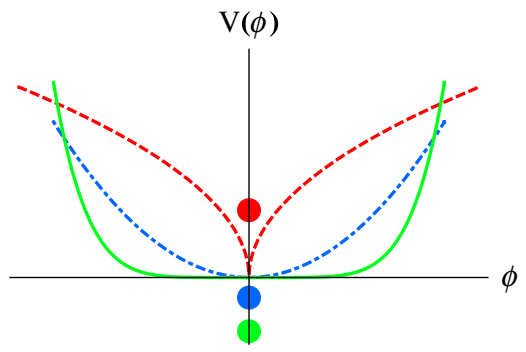

FIG. 1 (color online). Examples of potentials. The red dashed potential produce an equation-of-state corresponding to accelerated expansion $(w<-1 / 3)$. The average intercept for some amplitude is shown as the upper dot at $V>0$. The harmonic potential, which produces an equation-of-state parameter $w=0$, is the blue dot-dashed curve, and its average intercept the middle dot at $V<0$. Oscillations in the solid green potential produce an equation-of-state parameter $w>0$, with an average intercept at the lower dot at $V<0$. 
that the energy density must be potential dominated in order to produce accelerated expansion.

We also note that the sound speed (squared) $c_{s}^{2}$ is in general different than the equation-of-state parameter $w$, since the sound speed is given by [16]

$$
c_{s}^{2}=\frac{d(w \rho)}{\rho}=V_{0} \frac{d w}{d V_{0}}+w .
$$

We thus see that the sign of the sound speed is the same as the sign of $d T / d V_{0}$.

\section{HEURISTIC DISCUSSION OF DYNAMICAL INSTABILITY}

\section{A. Preview: the spintessence instability}

We begin by considering the growth of perturbations in spintessence. These models introduce a complex scalar field $\phi$ with a $U(1)$-symmetric potential $V(\phi)=V(|\phi|)$. The scalar field moves in a circular orbit in the potential at some constant amplitude $|\phi|$. There is kinetic energy associated with the spinning and potential energy associated with the displacement of the field from the minimum; the balance between the two is such that the equation-of-state parameter is $w=\left[|\phi| V^{\prime}(|\phi|)-\right.$ $2 V(|\phi|)] /\left[|\phi| V^{\prime}(|\phi|)+2 V(|\phi|)\right]$. For example, if $V(|\phi|) \propto|\phi|^{n}$, then $w=(n-2) /(n+2)$, matching the result for an oscillating real field.

Reference [3] showed (see also, Refs. [27]) that this coherently spinning field remains stable (neglecting gravity) to small perturbations if $V^{\prime}(|\phi|) /|\phi|-V^{\prime \prime}(|\phi|)<0$ and unstable, at sufficiently long wavelengths, if $V^{\prime}(|\phi|) /|\phi|-V^{\prime \prime}(|\phi|)>0$.

This result can be understood simply. The dynamics of perturbations in the linear regime are identical to the evolution of two particles undergoing circular orbits in a two-dimensional circularly symmetric potential $V(r)$; the gradient-energy density in the scalar field acts as a spring of force constant $k^{2}$ (where $k$ is the Fourier wave number of the perturbation) connecting the two particles. The radii of the two orbits differ initially by only a tiny amount. In the absence of any coupling between the two particles, they will evolve independently, spinning at two slightly different angular frequencies and each staying at its original amplitude. If $V^{\prime}(|\phi|) /|\phi|-V^{\prime \prime}(|\phi|)<0$, then the particle at larger $r$ will run slightly ahead, and if $V^{\prime}(|\phi|) /|\phi|-$ $V^{\prime \prime}(|\phi|)<0$, then it will run slightly behind.

Now suppose there is a very strong spring that attaches the two particles. In this case, the two particles will be bound to each other, and there will be no growth of perturbations. If there is a very weak spring, there will be energy transfer between the two particles on a time scale longer than the period of oscillation. In any confining potential, the angular momentum (per unit mass) increases as $r$ increases. If the particle at large $r$ runs ahead, as it will when $V^{\prime}(|\phi|) /|\phi|-V^{\prime \prime}(|\phi|)<0$, then it pulls the inner particle forward, donating some of its angular momentum in the process. In this way, the inner particle evolves to a slightly larger $r$ and the outer to a slightly lower $r$, decreasing their separation, and implying that the perturbation is stable. If, however, the particle at larger $r$ runs behind, as it will for $V^{\prime}(|\phi|) /|\phi|-V^{\prime \prime}(|\phi|)<0$, then it pulls on the inner particle, taking angular momentum from it. The inner particle must then evolve to a smaller- $r$ orbit and the outer particle to a larger- $r$ orbit. In this way, the small initial separation between the particles is amplified, and the perturbations are unstable. Readers familiar with accretiondisk physics will recognize this instability as the source of angular-momentum transport in disks (see, e.g., Ref. [28]).

For $V(|\phi|) \propto|\phi|^{n}$, the instability sets in for $n<2$. It is thus concluded that power-law spintessence potentials with negative pressure are subject to this instability and are therefore unsuitable as dark-energy candidates.

\section{B. The oscillating-field instability}

A similar argument can be applied to the growth of perturbations in oscillating-field matter. Adding a perturbation $\delta \phi(\vec{x}, t)$ to the homogeneous solution for the scalar field, the linear equation of motion for the perturbation obtained from Eq. (1) is

$$
\ddot{\delta \phi}-\nabla^{2} \delta \phi+V^{\prime \prime}(\phi) \delta \phi=0 .
$$

In linear theory, each Fourier mode of the density field $\delta \phi_{\vec{k}}$ evolves independently and satisfies an equation (suppressing the $\vec{k}$ subscript),

$$
\ddot{\delta \phi}+k^{2} \delta \phi+V^{\prime \prime}(\phi) \delta \phi=0 .
$$

This equation of motion is identical to that for the separation between two particles connected by a spring of force constant $k^{2}$ moving in a potential $V(\phi)$ with a separation $\delta \phi \equiv \phi_{2}-\phi_{1} \ll \phi_{1}, \phi_{2}$.

In this picture, particle 1 is released at rest from some initial height $\phi_{1} \sim \phi_{0}$ (where we will later identify $\phi_{0}$ as the amplitude of the background oscillations), and particle 2 is released slightly higher at $\phi_{2}=\phi_{1}+\delta \phi$. Because the frequency of oscillation will in general be amplitude dependent, if there is no spring connecting the two masses, then each oscillates at its own frequency. If $\omega^{\prime}\left(\phi_{0}\right)>0$, then the higher-amplitude mass (particle 2) runs ahead of the lower-amplitude mass (particle 1), and vice versa if $\omega^{\prime}\left(\phi_{0}\right)<0$.

If the spring connecting the two masses is extremely strong, then the two masses oscillate together. However, if the spring is weak, then energy can be exchanged between the two particles. If $\omega^{\prime}\left(\phi_{0}\right)>0$, then particle 2 runs ahead, pulls on particle 1 , and consequently donates some of its energy. Particle 1 then moves to a higher-amplitude orbit, particle 2 to a lower one, and the separation between them diminishes. Such perturbations are stable. If $\omega^{\prime}\left(\phi_{0}\right)<0$, then particle 1 runs ahead and donates energy to particle 2 . Particle 1 thus moves to a lower-amplitude orbit, and 


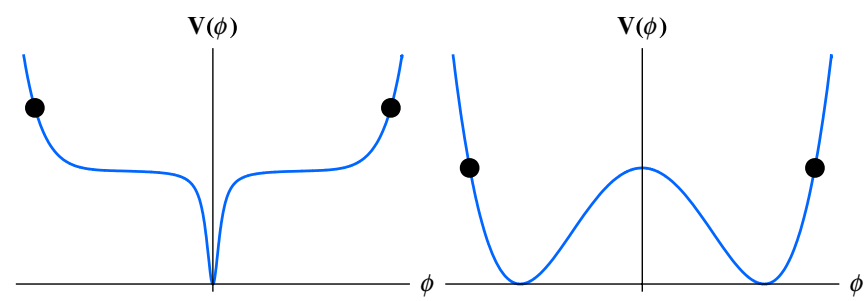

FIG. 2 (color online). Two examples of a potential that can produce accelerated expansion and have stability on small scales-but only with oscillation amplitudes near those indicated by the dots.

particle 2 to a higher orbit. In this case, the initial separation is amplified, and perturbations are unstable. Note that since this is a dynamical instability, the growth time scale will depend on the microphysical parameters of the potential, and will therefore typically be much faster than cosmological time scales.

We conclude that the sign of $\omega^{\prime}\left(\phi_{0}\right)$ provides a criterion for stability. This can be obtained from Eq. (7) as $\operatorname{sign}\left(\omega^{\prime}\right)=\operatorname{sign}\left(V_{0} d w / d V_{0}+w\right)$. As discussed above, power-law potentials with negative pressure have $\omega^{\prime}\left(\phi_{0}\right)<0$, and so will develop large-scale (small $k$ ) instabilities, rendering these models unsuitable for accounting for dark energy. Using the geometrical condition Eq. (8) as guidance, it is possible to find potentials that produce cosmological acceleration and do not develop large-scale instabilities, but only for a small range of amplitudes. The potential in the left cell of Fig. 2, which is of the form suggested by Ref. [16], will exhibit longterm cosmic acceleration, but only short-term stability as the amplitude of oscillation inevitably decays. The potential in the right cell of Fig. 2 will exhibit long-term stability, but only short-term cosmic acceleration. The analysis of the viability of these models is somewhat more involved, although a significant and perhaps unnatural tuning of both the initial conditions and the potential seem necessary.

Of course, care must be taken with the above arguments, as the energy exchange between the particles must be considered throughout the particles' orbits, and not just at the outset of their motion. Nevertheless, as we shall discuss further below, we have been able to verify analytically that perturbations in nearly-harmonic potentials (to be defined more precisely below) with $\omega^{\prime}\left(\phi_{0}\right)<0$ are unstable, while those with $\omega^{\prime}\left(\phi_{0}\right)>0$ are stable. We will then discuss numerical results that support these conclusions.

\section{QUANTITATIVE ANALYSIS OF DYNAMICAL INSTABILITY}

We now return to Eq. (11) to discuss the quantitative behavior of perturbations. In a harmonic potential, $V^{\prime \prime}=$ $\omega^{2}$ is constant, and $\delta \phi$ oscillates with fixed amplitude for all $k^{2}$. In the most general potential, $V^{\prime \prime}(\phi)$ oscillates- although not necessarily sinusoidally - with an oscillation frequency $2 \omega$, and so the perturbation equation, Eq. (11) is that of a harmonic oscillator with an oscillating mass.

The solutions to Eq. (11), as well as the issue of their stability, is the subject of the Floquet theory. ${ }^{1}$ There is no simple stability condition in the most general case, but we can obtain an analytic solution for potentials that are close to harmonic. To begin, consider the quartic potential $V(\phi)=(1 / 2) m^{2} \phi^{2}+(\lambda / 4) \phi^{4}$, working to linear order in $\lambda$ in the limit $\lambda \rightarrow 0$. In this case, $V^{\prime \prime}(\phi)=m^{2}+$ $3 \lambda \phi^{2}$. If the oscillation amplitude is $\phi_{0}$, the oscillation frequency is $\omega^{2}=m^{2}\left[1+(3 / 4) \lambda \phi_{0}^{2} / m^{2}\right]$ and the homogeneous oscillation is nearly sinusoidal: $\phi(t)=\phi_{0} \cos \omega t$. With the trigonometric identity, $\cos ^{2} x=(1 / 2) \times$ $(1+\cos 2 x)$, the perturbation equation is, to linear order in $\lambda$, then

$\ddot{\delta} \phi+\left\{\left[k^{2}+m^{2}+(3 / 2) \lambda \phi_{0}^{2}\right]+(3 / 2) \lambda \phi_{0}^{2} \cos 2 \omega t\right\} \delta \phi=0$.

Defining $z=\omega t$, this is identified as the Mathieu equation,

$$
\frac{d^{2}}{d z^{2}} \delta \phi+[a-2 q \cos 2 z] \delta \phi=0,
$$

with

$$
\begin{aligned}
& a=\left(1+\frac{k^{2}}{m^{2}}\right)\left(1-\frac{3}{4} \frac{\lambda \phi_{0}^{2}}{m^{2}}\right)+\frac{3}{2} \frac{\lambda \phi_{0}^{2}}{m^{2}}, \\
& q=-\frac{3}{4} \frac{\lambda \phi_{0}^{2}}{m^{2}} .
\end{aligned}
$$

If we consider only long-wavelength (i.e., $k \ll m$ ) fluctuations and the limit $\lambda \phi_{0}^{2} \ll m^{2}$, then $a \simeq 1$ and $q \ll 1$. In this regime, $\delta \phi$ oscillates rapidly with frequency $\omega t$ with an amplitude that varies as $e^{ \pm \Omega t}$ with $\Omega \simeq$ $\sqrt{q^{2}-(1-a)^{2}} \omega$. Thus, the condition for instability is $1-$ $|q|<a<1+|q|$ (as derived in Sec. VI below).

Thus, if $\lambda>0$, then the solutions are stable for all $k^{2}>$ 0 . For $\lambda<0$, the solutions are stable only if $k^{2}>k_{J}^{2}=$ $-(3 / 2) \lambda \phi_{0}^{2}$. Recalling that the oscillation frequency is $\omega^{2} \simeq m^{2}+3 \lambda \phi_{0}^{2}$, we see that there is instability, on sufficiently large scales $k^{-1}$, if $\omega^{\prime}\left(\phi_{0}\right)<0$, while the perturbations are stable for all $k$ if $\omega^{\prime}\left(\phi_{0}\right)>0$.

We can also generalize to other potentials that are close to harmonic, by which we mean that the time dependence of $V^{\prime \prime}[\phi(t)]$ can be approximated as $V^{\prime \prime}(t) \approx V_{0}^{\prime \prime}+$ $V_{2}^{\prime \prime} \cos 2 \omega t$ with constant $V_{0}^{\prime \prime}$ and $V_{2}^{\prime \prime}$. The Jeans wave number $k_{J}$ is then given by

$$
k_{J}^{2}=\frac{1}{2}\left|V_{2}^{\prime \prime}\right|+\omega^{2}-V_{0}^{\prime \prime} .
$$

This will typically be much smaller than cosmological

\footnotetext{
${ }^{1}$ If we replace time $t$ by a position $x$, our equation becomes the Schrodinger equation for a particle in a periodic potential, the solutions of which are described by Bloch's theorem.
} 
distance scales. It can be checked numerically that for the power-law potentials $V(\phi) \propto|\phi|^{n}$ with $n \simeq 2$, these relations imply stability for $n>2$ and instability for $n<2$, as our heuristic arguments suggest.

Of course, the types of potentials that can drive cosmic acceleration are far from harmonic (for the power-law potential, an index $n<1$ is required), and so the analysis presented above will no longer be valid. However, we expect no qualitative difference, and to confirm this we investigate the stability of oscillations in more complicated potentials numerically in the next section.

\section{NUMERICAL RESULTS ON DYNAMICAL INSTABILITY}

We will numerically determine the stability of oscillations in the presence of three different representative classes of potential in this section: power-law potentials with arbitrary index, temporarily stable potentials exhibiting cosmic acceleration (as in the left cell of Fig. 2), and stable potentials exhibiting temporary cosmic acceleration (as in the right cell of Fig. 2). In each case, we write the potential in the form $V(\phi)=\mu^{4} v\left(\phi / \phi_{0}\right)$, and define the dimensionless variables,

$$
x=\phi / \phi_{0}, \quad \tau=\frac{\mu^{2}}{\phi_{0}} t, \quad \kappa=\frac{\phi_{0}}{\mu^{2}} k, \quad y=\frac{\delta \phi}{A},
$$

where $A$ is an appropriately defined constant characterizing the amplitude of the perturbation. The equations of motion then become

$$
0=\frac{d^{2} x}{d \tau^{2}}+\frac{d v}{d x}, \quad 0=\frac{d^{2} y}{d \tau^{2}}+\left[\kappa^{2}+\frac{d^{2} v}{d x^{2}}\right] y .
$$

To determine the regions of stability, we can form the fundamental solution matrix (for a more detailed discussion of determining the stability of Hill's equation, see, e.g., Ref. [29])

$$
C=\left(\begin{array}{cc}
y_{1}(T / 2) & y_{2}(T / 2) \\
d y_{1} / d \tau(T / 2) & d y_{2} / d \tau(T / 2)
\end{array}\right),
$$

where $y_{1}$ is the solution generated from the initial conditions $\{y(0)=1, d y / d \tau(0)=0\}$, and $y_{2}$ is the solution generated from the initial conditions $\{y(0)=0, d y / d \tau(0)=1\}$. The stability of a solution can be determined by the eigenvalues of $C$ [noting that $\operatorname{det}(C)=1$ ]

$$
\Lambda_{ \pm}=\frac{\operatorname{tr}(C) \pm \sqrt{\operatorname{tr}(C)^{2}-4}}{2} .
$$

Instability results when either eigenvalue has modulus greater than unity. Since $\Lambda_{+} \Lambda_{-}=1$ (from the determinant condition), if the roots are both real, then solutions are unstable. This occurs when $|\operatorname{tr}(C)|>2$. It also follows that on the boundary between stability and instability, $y_{1}(t)$ is a function of period $T / 2$ or $T$ (either the same period as $v^{\prime \prime}$ or $x$ ). Because the system of equations we are studying are invariant under time shifts by a period of oscillation, from $y_{1}(0)=d y_{2} / d \tau(0)=1$, we will have $y_{1}(T / 2)=$ $d y_{2} / d \tau(T / 2)$. The stability criterion can in this case be phrased as $\left|y_{1}(T / 2)\right|<1$, and provides an easy way to numerically determine stability or instability.

We will first consider power-law potentials

$$
V(|\phi|)=\lambda|\phi|^{n} .
$$

The singularity at the origin can be regulated by introducing a numerically small smoothing parameter $c$, such that $V(|\phi|)=\lambda\left(c+\phi^{2}\right)^{n / 2}$. We have checked that the numerics are insensitive to the value of this parameter. Numerically integrating Eq. (17), and solving for the roots of $\left|y_{1}(T / 2)\right|-1$, we can find the boundaries of stability. Plotted in Fig. 3 are the seven lowest bands of instability (the shaded regions of the figure) for power-law potentials with $n<3$. It can be seen that there is a band of instability encompassing $k=0$ for $n<2$ as expected from the general arguments given above. In addition, as $n$ decreases, the higher-order bands of instability migrate towards a lower wave number. For power-law potentials with an equationof-state parameter $w \sim-1$, the power-law index will be rather small, and the large number of higher-order bands of instability at small $k$ might become important in analyzing the overall stability of such a model.

Moving to potentials of the form shown in the left cell of Fig. 2, which we can write as,

$$
V(|\phi|)=\mu^{4}\left[\frac{x^{2}}{c_{1}+x^{2}}+c_{2} x^{n}\right]
$$

we chose as an example $c_{1}=c_{2}=0.005$ and $n=10$. Again, numerically integrating Eq. (17), we plot the first six bands of instability in Fig. 4. Also overplotted are the values of the equation-of-state parameter corresponding to various amplitudes of the background oscillations. It can be seen that there are indeed regions where $w<-1 / 3$ and large-scale stability exists. However, as the universe evolves and the amplitude of oscillation decays, more

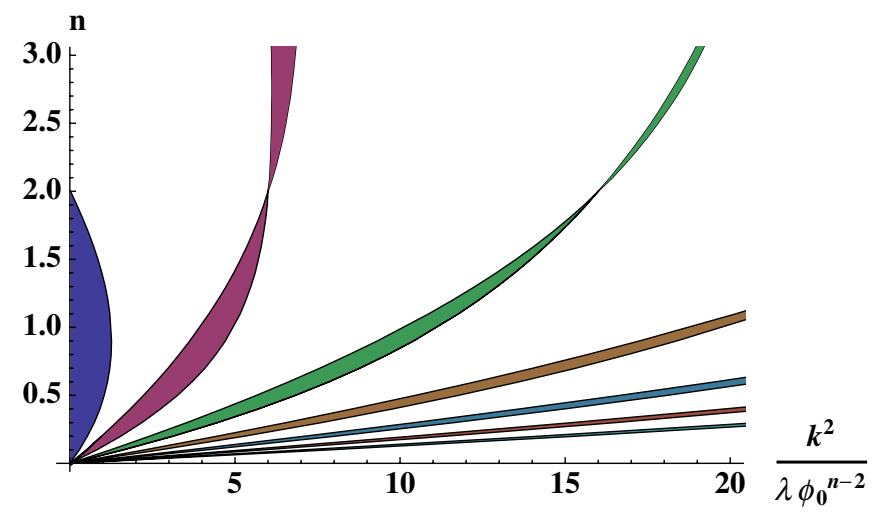

FIG. 3 (color online). The first seven bands of instability for oscillations in the presence of a power-law potential. 


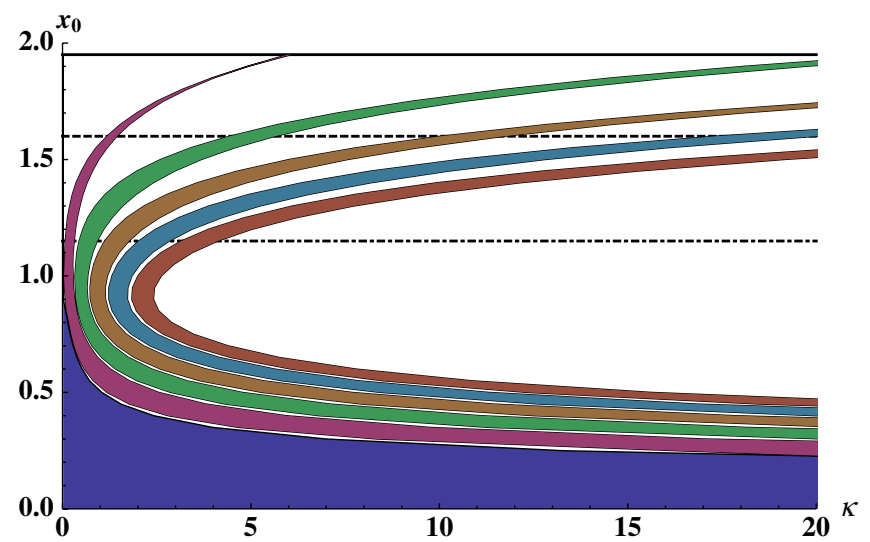

FIG. 4 (color online). The first six bands of instability for oscillations in the presence of the potential shown in the left cell of Fig. 2. The solid line represents an amplitude corresponding to $w=-0.4$, the dashed line $w=-0.6$, and the dot-dashed line $w=-0.8$. In this example, $w$ remains less than one, but as the amplitude of oscillations decays a large-scale band of instability develops.

and more bands of instability will pile up at small $k$. When the amplitude of oscillation dips below $x_{0} \sim 1$ in this model, the solution will become violently unstable at both small and large $k$, with only very small bands of stability. This will most likely present a severe challenge to using such models as a candidate for the dark energy. We have also checked numerically that the large-scale (encompassing $k=0$ ) band of instability appears when the amplitude of oscillation is such that $w^{\prime}$ changes sign, supporting, in a more complicated potential, our heuristic arguments for stability.

Finally, we will treat the case where we expect the potential to exhibit large-scale stability, but only temporary cosmic acceleration, as in the right cell of Fig. 2. The potential is given by

$$
V(\phi)=\mu^{4}\left(x^{2}-c^{2}\right)^{2},
$$

where we choose as an example $c=1$. When the amplitude is very close to the height of the barrier between the two minima of this potential (at $x_{0}=\sqrt{2}$ ), we expect the equation-of-state parameter to be $w \sim-1$. $^{2}$ As shown in Fig. 5, this potential yields large-scale stability, with the first order band of instability approaching $k=0$ as the amplitude of oscillations reaches $x_{0} \sim \sqrt{2}$. Also shown are the values of $w$ for a number of amplitudes.

In all of our examples, we have verified numerically that the sign of $\omega^{\prime}$ determines the existence of a band of

\footnotetext{
${ }^{2}$ The field will loiter near the local maximum, extending the period of oscillations, and eventually spoiling our approximation that the period be shorter than a Hubble time; we will ignore this shortcoming for the moment. We also note that when quantum effects are included, there will exist additional forms of instability [30], which can lead to the rapid decay of the background oscillations.
}

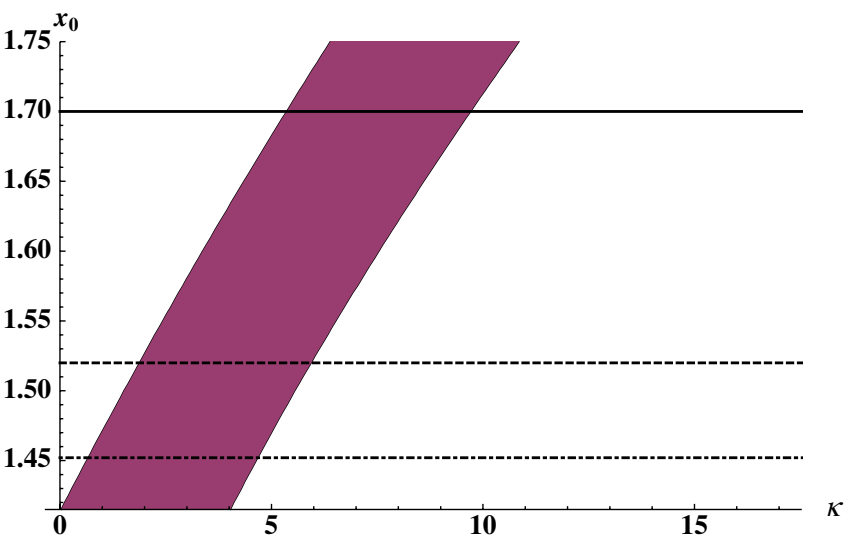

FIG. 5 (color online). The instability band for oscillations in the double-well potential shown in the right cell of Fig. 2. There is just one band of instability until $k$ becomes very large. Also plotted are lines that indicate the amplitudes at which $w=0.4$, $w=0.2$, and $w=0$ (from top to bottom). Note that $w \rightarrow-1$ as $x \rightarrow \sqrt{2} \simeq 1.41$, and so this model features large-scale stability with $-1<w<0$. However, this period of negative pressure is short lived as the Universe expands.

instability about $k=0$, as expected from the heuristic arguments of Sec. III B. We have also illustrated the existence of higher-order bands of instability that will influence the development of small-scale inhomogeneities in the oscillating field. In all examples, the higher bands of instability creep in towards $k=0$ as $w \rightarrow-1$.

\section{GRAVITATIONAL INSTABILITY}

\section{A. General analysis}

We now include gravity in the analysis. As alluded to above, perturbations in a perfect fluid with sound speed $c_{s}$ are stabilized by pressure gradients for wave numbers $k>$ $k_{J}=\left(4 \pi G \rho / c_{s}^{2}\right)^{1 / 2}$, but care must be taken in applying this result to coherent scalar fields. For example, oscillations in a harmonic potential have a sound speed $c_{s}^{2}=$ $d(w \rho) / d \rho=0$, implying that perturbations on all scales will suffer a gravitational instability. However, as shown in Ref. [25], perturbations on scales $k>2 \sqrt{\pi G} m^{2} \phi_{0}$ are stabilized. We therefore extend the analysis of Ref. [25] to see how their result for the scalar-field stability is altered by the presence of a small anharmonic term in the potential. This is in fact general, since, as we will see, strong dynamical instabilities will completely swamp the gravitational instability, implying that the inclusion of gravitational effects is only relevant for potentials that are close to harmonic (as we have defined above).

To simplify the analysis, we restrict our attention to small-wavelength Fourier modes, $k \gg H$, where $H=$ $(8 \pi G \rho / 3)^{1 / 2}$ and $\rho=(1 / 2) \dot{\phi}^{2}+V(\phi)$. This allows us to neglect the expansion and work with a perturbed Minkowski metric (in the conformal Newtonian gauge),

$$
d s^{2}=(1+2 \Psi) d t^{2}+(1-2 \Psi) d \vec{x}^{2},
$$


where $\Psi$ is the Newtonian potential. Perturbations $\delta \phi$ in the scalar field may now induce perturbations $\Psi$ to the metric, which then affect the scalar-field equation of motion, which is now,

$$
\ddot{\delta \phi}+\left[V^{\prime \prime}(\phi(t))-\nabla^{2}\right] \delta \phi=4 \dot{\Psi}(\dot{\delta \phi})-2 \Psi V^{\prime}(\phi(t)) \text {. }
$$

The potential is determined by the Einstein equation (the Poisson equation),

$$
\nabla^{2} \Psi=4 \pi G\left[\dot{\phi}(\dot{\delta \phi})+V^{\prime}(\phi(t)) \delta \phi-\Psi \dot{\phi}^{2}\right] .
$$

We now focus on a given Fourier mode of wave number $\vec{k}$, in which case we replace $\nabla^{2} \rightarrow-k^{2}$ in these equations, and we neglect the last term on the right-hand side of Eq. (25), as it is negligible for $k \gg H$.

Although the equations have become more complicated with gravity, they are once again linear differential equations with periodic coefficients, and so the solutions are formally described by Floquet theory.

\section{B. A quartic correction to a harmonic potential}

We now consider the potential $V(\phi)=(1 / 2) m^{2} \phi^{2}+$ $(\lambda / 4) \phi^{4}$ and suppose that the anharmonic term is small, $\lambda \phi_{0}^{2} \ll m^{2}$. Equations (24) and (25) can then be combined into a single second-order differential equation,

$$
\begin{aligned}
\ddot{\delta} \phi+ & m \gamma \sin 2 \omega t \dot{\delta} \phi+\left[k^{2}+m^{2}+\frac{3}{2} \lambda \phi_{0}^{2}\right. \\
& \left.-\gamma m^{2}+\left(\frac{3}{2} \lambda \phi_{0}^{2}-\gamma m^{2}\right) \cos 2 \omega t\right] \delta \phi=0,
\end{aligned}
$$

where $\gamma \equiv G \phi_{0}^{2} / k^{2}$. At the lowest- $k$ stability boundary, the solutions are periodic with frequency $\omega$, and can thus be written $\delta \phi(t)=a \cos \omega t+b \sin \omega t$. We then plug this into Eq. (26) and demand that the coefficients of the terms that vary as $\cos \omega t$ and as $\sin \omega t$ vanish. In doing so, we work to linear order in $\lambda$ and $\gamma$ and neglect terms of $O(\lambda \gamma)$. We then find that the boundary between stability and instability occurs for wave numbers $k_{1}$ and $k_{2}$ given by

$$
k_{1}^{2}=-\frac{3}{2} \lambda \phi_{0}^{2}+\gamma m^{2}, \quad k_{2}^{2}=0 .
$$

A bit more algebra [replacing $a$ and $b$ by slowly varying functions $a(t)=a_{0} \exp (\Omega t)$ and $b(t)=b_{0} \exp (\Omega t)$, with $\Omega \ll \omega]$ shows that the instability occurs for values of $k$ between $k_{1}$ and $k_{2}$.

If $\lambda<0$, then $k_{1}^{2}>0$. In this case, the field is dynamically unstable, even without gravity (as noted before), and gravity only serves to increase the instability. If, however, $\lambda>0$, gravity induces an instability (noting that $\gamma \propto k^{-2}$ ), for $k^{2}<k_{J}^{2}$, with

$$
k_{J}^{2}=-\frac{3}{4} \lambda \phi_{0}^{2}+\sqrt{\left(\frac{3}{4} \lambda \phi_{0}^{2}\right)^{2}+8 \pi G m^{4} \phi_{0}^{2}} .
$$

Note that this recovers our earlier result, without gravity, in the limit that $G \rightarrow 0$, and it recovers the result of Refs. [25,26] for $\lambda=0$.
The result for the Jeans scale resembles that for spintessence [cf., Eq. (10) in Ref. [3]] with the replacement $(1 / 2)\left(V^{\prime} /|\phi|-V^{\prime \prime}\right)=-\lambda\left|\phi_{0}^{2}\right|$ for spintessence with $-(3 / 4) \lambda \phi_{0}^{2}$ for the oscillating field, and replacing the $G$ in the spintessence result by $G / 2$. The former replacement occurs because the sound speed $c_{s}^{2}=d(w \rho) / d \rho=$ $(1 / 2) \lambda\left(\phi_{0}^{2} / \mathrm{m}^{2}\right)$ for spintessence differs from that, $c_{s}^{2}=$ $(3 / 8) \lambda\left(\phi_{0}^{2} / \mathrm{m}^{2}\right)$, for the oscillating field. The latter replacement (i.e., $G \rightarrow G / 2$ ) occurs because the complex field is equivalent to two scalar fields. Note that the result, Eq. (28), differs from the spintessence result also because the spintessence Jeans scale of Eq. (10) in Ref. [3] places no restrictions on the potential $V(R)$, while Eq. (28) here is valid only in the limit $\lambda \phi_{0}^{2} \ll m^{2}$.

In the limit that $G m^{4} \phi_{0}^{2} \ll \lambda^{2} \phi_{0}^{4}, k_{J}^{2} \simeq 16 \pi G m^{4} / 3 \lambda$, which is equal to $4 \pi G / c_{s}^{2}$, the Jeans scale for a perfect fluid. However, if $G m^{4} \phi_{0}^{2} \gg \lambda^{2} \phi_{0}^{4}$, then the perfect-fluid description breaks down, the scalar-field dynamics become important, and the Jeans length differs considerably from the perfect-fluid result, $c_{s}^{2} k_{J}^{2}=4 \pi G \rho$.

\section{General result for nearly-harmonic potentials}

The result, Eq. (28), can be generalized to other potentials that are close to harmonic by replacing $-(3 / 2) \lambda \phi_{0}^{2}$ in Eq. (28) by $(1 / 2)\left|V_{2}^{\prime \prime}\right|+\omega^{2}-V_{0}^{\prime \prime}$,

$$
\begin{aligned}
k_{J}^{2}= & \frac{1}{2}\left[\frac{1}{2}\left|V_{2}^{\prime \prime}\right|+\omega^{2}-V_{0}^{\prime \prime}\right. \\
& \left.+\sqrt{\left[\frac{1}{2}\left|V_{2}^{\prime \prime}\right|+\omega^{2}-V_{0}^{\prime \prime} \mid\right]^{2}+8 \pi G m^{4} \phi_{0}^{2}}\right] .
\end{aligned}
$$

\section{Application: Axion dark matter}

We now calculate the small-scale cutoff in the colddark-matter power spectrum under the assumption that the dark matter is composed of axions with masses $m_{a} \sim$ $10^{-5} \mathrm{eV}$ [31]. This cutoff will determine the masses of the first dark-matter halos to undergo collapse in the Universe, and it determines the size of the smallest clumps in the Milky Way halo [32,33]. If weakly-interacting massive particles (WIMPs) make up the dark matter, then the primordial power spectrum is suppressed for cosmological mass scales smaller than $\sim 10^{-4}-10^{2} M_{\oplus}$ (the precise value determined by the precise WIMP model) [34] by kinetic decoupling of WIMPs. We will now calculate the analogous small-scale cutoff if axions make up the dark matter.

Cosmological axions in the $\sim 10^{-5} \mathrm{eV}$ mass regime are produced by the misalignment mechanism near the time of the QCD phase transition, and they may thus be described by a coherently oscillating scalar field. The axion potential has the form $V(\phi)=V_{0}[\cos (\phi / f)-1]$. Today, the axion field oscillates near the minimum of this potential where it can be approximated by $V(\phi) \approx(1 / 2) m^{2} \phi^{2}+(\lambda / 4) \phi^{4}$, with $m^{2}=V_{0} / f^{2}$ and $\lambda=-(1 / 6)\left(m^{2} / f^{2}\right)$. Here, $f \simeq$ $\Lambda^{2} / m$ is the Peccei-Quinn scale, where $\Lambda \sim 100 \mathrm{MeV}$ is 
the QCD scale. If axions make up the dark matter, then the axion energy density today is $\rho_{q}=(1 / 2) m^{2} \phi_{0}^{2}=\Omega_{c} \rho_{c}$, where $\Omega_{c} \simeq 0.2$ is the cold-dark-matter density and $\rho_{c}$ is the critical density, and this relation can be used to fix $\phi_{0}$. Using the scaling $\phi_{0}^{2} \propto(1+z)^{3}$, we find also that $\left(\lambda \phi_{0}^{2}\right)^{2} \ll G m^{4} \phi_{0}^{2}$ not only today, but at all redshifts $z \lessgtr$ 3000 during matter domination. The axion-axion interactions implied by the $\lambda \phi^{4}$ correction to the quadratic potential therefore have little effect on the Jeans scale, which is well approximated by the earlier result of Refs. [25,26].

The physical Jeans scale is given by $k_{J}^{2}=2 \sqrt{2 \pi G} m^{2} \phi_{0}$, and the Jeans mass then turns out to be $M_{J} \equiv(4 \pi / 3) \times$ $\left(\pi / k_{J}\right)^{3} \rho_{m}$, where $\rho_{m}$ is the current matter density. Numerically,

$$
M_{J} \simeq 1.8 \times 10^{-13}\left(m_{a} / 10^{-5} \mathrm{eV}\right)^{-3 / 2}(1+z)^{-3 / 4} M_{\oplus} .
$$

The collapse redshift for such low-mass halos depends on the primordial spectral index, but is generally in the range $3000 \gtrsim z \gtrsim 300$, resulting in a small-scale cutoff of order $10^{-15} M_{\oplus}$ in the primordial power spectrum, much smaller than that for WIMPs. Note that there may also be dynamical instabilities on even smaller scales (related to the higher-order bands of instability, as in Sec. V); see, e.g., the very elegant work of Ref. [35].

\section{CONCLUSIONS}

We have considered the dynamical and gravitational amplification of inhomogeneities in oscillating-field matter. We provided a simple physical picture for the origin of instabilities in oscillating-field matter and in spintessence. In this picture, the condition for instability is seen as a condition on the amplitude dependence of the frequency, a condition that can be shown to be equivalent to positivity of the sound speed. This argument was verified analytically for nearly-harmonic potentials and numerically for more general potentials. We then included gravity in the analysis, generalizing earlier results on the gravitational insta- bility of harmonic potentials to potentials that differ slightly from harmonic. We used this result to evaluate the small-scale cutoff in the matter power spectrum if axions make up the dark matter. We leave the implications of this cutoff (roughly $10^{-15} M_{\oplus}$ ) to future work.

Our results indicate that potentials that give rise to accelerated expansion generically suffer dynamical instabilities to the growth of large-scale inhomogeneities. These instabilities should render oscillating fields unsuitable to account for dark energy in the Universe today or for driving inflation in the early Universe, as both scenarios require the cosmological density to remain homogeneous for extended periods of time. It is true that there may be potentials that drive acceleration and are stable, but stability and/or acceleration will be only temporary. This loophole is thus unlikely to alter our conclusions.

In order to simplify the instability analysis, we have restricted our attention to modes with wavelengths small compared with the Hubble scale and modes with growth rates that are much larger than the Hubble time. In this limit, the growth of perturbations will be exponential. It may well be, however, that a more complete analysis would extend the bands of instability to time scales/wavelengths comparable to the Hubble time/distance. If so, the growth of perturbations may be slowed, relative to exponential and the growth may be limited by the shifting of the unstable wavelength out of a band of instability [36].

We were able to make progress analytically for both the dynamical and gravitational instability only for nearlyharmonic potentials. It would be interesting to see whether analytic results for the growth of perturbations can be extended to more general potentials. We leave the investigation of this question for future work.

\section{ACKNOWLEDGMENTS}

We thank D. Cohen for discussions of Floquet theory, L. Kofman for suggesting some useful papers, and C. Hirata for discussions and suggestions. This work was supported by the DOE DE-FG03-92-ER40701 and the Gordon and Betty Moore Foundation.
[1] S. Perlmutter et al. (Supernova Cosmology Project Collaboration), Astrophys. J. 517, 565 (1999); A. G. Riess et al. (Supernova Search Team Collaboration), Astron. J. 116, 1009 (1998).

[2] B. Ratra and P.J.E. Peebles, Phys. Rev. D 37, 3406 (1988); C. Wetterich, Astron. Astrophys. 301, 321 (1995); K. Coble, S. Dodelson, and J. A. Frieman, Phys. Rev. D 55, 1851 (1997); M. S. Turner and M. J. White, Phys. Rev. D 56, R4439 (1997); R. R. Caldwell, R. Dave, and P. J. Steinhardt, Phys. Rev. Lett. 80, 1582 (1998).
[3] L. A. Boyle, R. R. Caldwell, and M. Kamionkowski, Phys. Lett. B 545, 17 (2002).

[4] J. A. Gu and W. Y. Hwang, Phys. Lett. B 517, 1 (2001).

[5] M. S. Turner, Phys. Rev. D 28, 1243 (1983).

[6] T. Damour and V.F. Mukhanov, Phys. Rev. Lett. 80, 3440 (1998).

[7] A. R. Liddle and A. Mazumdar, Phys. Rev. D 58, 083508 (1998).

[8] J. w. Lee, S. Koh, C. Park, S. J. Sin, and C. H. Lee, Phys. Rev. D 61, 027301 (1999). 
[9] V.H. Cardenas and G. Palma, Phys. Rev. D 61, 027302 (1999).

[10] M. Sami, Gravitation Cosmol. 8, 309 (2003).

[11] V. Sahni, M. Sami, and T. Souradeep, Phys. Rev. D 65, 023518 (2001).

[12] A. R. Liddle and R. J. Scherrer, Phys. Rev. D 59, 023509 (1998).

[13] M. Sami, N. Dadhich, and T. Shiromizu, Phys. Lett. B 568, 118 (2003).

[14] L. Perivolaropoulos, Phys. Rev. D 67, 123516 (2003).

[15] V. Sahni and L. M. Wang, Phys. Rev. D 62, 103517 (2000).

[16] E. Masso, F. Rota, and G. Zsembinszki, Phys. Rev. D 72, 084007 (2005).

[17] J.a. Gu, arXiv:0711.3606.

[18] S. D. H. Hsu, Phys. Lett. B 567, 9 (2003).

[19] S. Dutta and R. J. Scherrer, arXiv:0805.0763.

[20] M. Sami and T. Padmanabhan, Phys. Rev. D 67, 083509 (2003); 67, 109901 (2003).

[21] S. Tsujikawa, Phys. Rev. D 61, 083516 (2000).

[22] A. Taruya, Phys. Rev. D 59, 103505 (1999).

[23] S. Kasuya, M. Kawasaki, and F. Takahashi, Phys. Lett. B 559, 99 (2003).

[24] S. Kasuya, Phys. Lett. B 515, 121 (2001).

[25] M. Khlopov, B. A. Malomed, and I. B. Zeldovich, Mon. Not. R. Astron. Soc. 215, 575 (1985).

[26] W. Hu, R. Barkana, and A. Gruzinov, Phys. Rev. Lett. 85,
1158 (2000).

[27] A. Kusenko and M.E. Shaposhnikov, Phys. Lett. B 418, 46 (1998); K. Enqvist and J. McDonald, Nucl. Phys. B38, 321 (1999); S. Kasuya, Phys. Lett. B 515, 121 (2001).

[28] J. Frank, A. King, and D. Raine, Accretion Power in Astrophysics (Cambridge University Press, Cambridge, 2002).

[29] W. Magnus and S. Winkler, Hill's Equation (Interscience Publishers, New York, 1966).

[30] G. N. Felder, L. Kofman, and A. D. Linde, Phys. Rev. D 64, 123517 (2001).

[31] M.S. Turner, Phys. Rep. 197, 67 (1990); G. G. Raffelt, Phys. Rep. 198, 1 (1990); L. J. Rosenberg and K. A. van Bibber, Phys. Rep. 325, 1 (2000); S. J. Asztalos, L. J. Rosenberg, K. van Bibber, P. Sikivie, and K. Zioutas, Annu. Rev. Nucl. Part. Sci. 56, 293 (2006).

[32] J. Diemand, B. Moore, and J. Stadel, Nature (London) 433, 389 (2005).

[33] M. Kamionkowski and S. M. Koushiappas, Phys. Rev. D 77, 103509 (2008).

[34] S. Profumo, K. Sigurdson, and M. Kamionkowski, Phys. Rev. Lett. 97, 031301 (2006).

[35] P. B. Greene, L. Kofman, and A. A. Starobinsky, Nucl. Phys. B43, 423 (1999).

[36] Y. Shtanov, J. H. Traschen, and R. H. Brandenberger, Phys. Rev. D 51, 5438 (1995). 